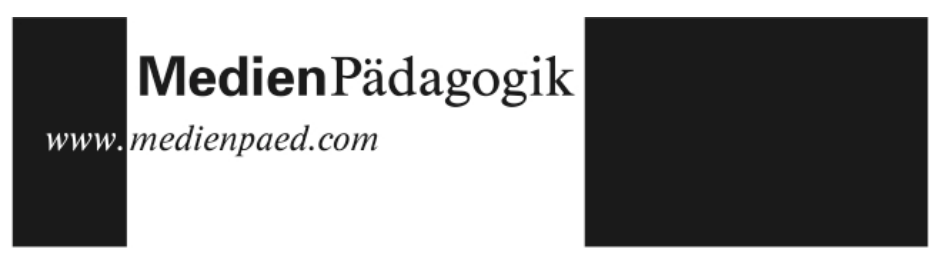

Rezensionen

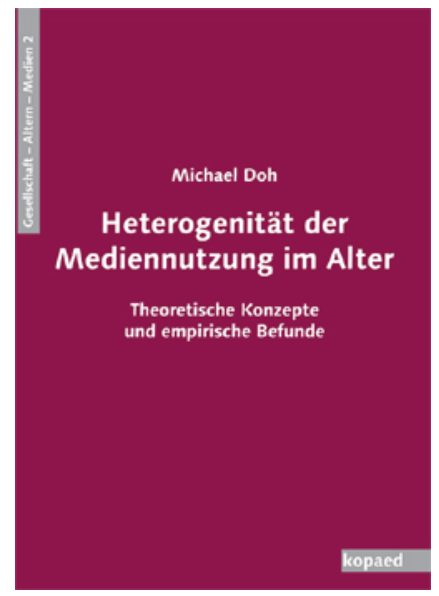

\section{Seniorenwelten sind Fernsehwelten}

In Abwandlung des in den 1980er Jahren von Uwe Sander und Ralf Vollbrecht postulierten Slogans «Kinderwelten sind Medienwelten» könnte die gewählte Überschrift die Essenz aus der von Michal Doh verfassten Dissertation «Heterogenität der Mediennutzung im Alter» sein. Während vor mehr als 20 Jahren die Inklusion des Fernsehens in die Lebenswelt Heranwachsender im Fokus des wissenschaftlichen Interesses stand, gilt dieses nunmehr auch für Erwachsene im höheren Alter. Der Autor kommt in seinen Analysen zum Schluss, dass sich der Aktionsradius älterer Menschen zunehmend auf das häusliche Umfeld fokussiert und das genutzte Medienensemble sich verkleinert. Das Leitmedium Fernsehen hat hier einen besonderen Stellenwert, weshalb Doh dieses in den Mittelpunkt seiner Arbeit stellt. Ein zentrales Anliegen ist inm dabei die (bislang kaum praktizierte) Verbindung theoretischer Konzepte und Methoden aus Gerontologie und Kommunikations- und Medienwissenschaft. Mit dem Ziel einer integralen Verknüpfung führt er Erkenntnisse beider Wissenschaftsfelder systematisch zusammen.

Nach seinem einleitenden Kapitel, das diese Zielsetzung begründet, setzt sich Doh im zweiten Kapitel zunächst mit den theoretischen Grundlagen und empirischen Befunden zum Themenkomplex Altern und Medien auseinander. Er systematisiert das interdisziplinäre Forschungsfeld aus gerontologischen, medienwissenschaftlichen, soziologischen, sozio-ökologischen, philosophischen und anthropologischen Zugängen anhand einer von ihm entworfenen und sehr gut nachvollziehbaren Ordnungsstruktur in individuum-, umwelt- und gesellschaftsbezogenen Theorieaspekten. 
Ergänzende Sichtweisen zum Thema Altern und Medien, die sich dieser Logik nicht zuordnen lassen aber dennoch von Relevanz sind, werden in einem gesonderten Abschnitt aufgegriffen. Zusammenfassend konstatiert Doh aus Theorie und Empirie zentrale Forschungsdesiderata: So fehlen noch immer empirische Befunde, die eine differenzierte Betrachtung der Mediennutzung älterer Menschen erlauben und kontextbezogene Faktoren (personen- und umweltbezogen) in sich verändernden Lebenswelten systematisch einbeziehen.

Seine eigene Forschungsmethodologie integriert - ableitend aus seiner erkenntnistheoretischen Perspektive - einen medienzentrierten und einen gerontologischen Analysestrang. Dabei geht er sekundäranalytisch vor, indem auf die Erhebungswellen der Langzeitstudie Massenkommunikation (MK) der ARD/ZDF Medienkommission aus den Jahren 2000 und 2005 zurückgreift. Konkret wird die kontextuelle Untersuchung der Mediennutzung älterer Menschen nach den Kategorien Alter, Kohorte, Periode sowie intrakohortenspezifische Heterogenität entlang soziodemographischer Merkmale bei den 1930-1939 geborenen analysiert. Der zweite Teil des Forschungsdesigns bildet die vertiefende und explorative Untersuchung der Kohorte 1930-1932 innerhalb des dritten Messzeitpunktes (2005) der «Interdisziplinäre Längsschnittstudie des Erwachsenenalters» (ILSE). Hier erstellt der Autor eine Typologie von Nutzungsmotiven anhand des Leitmediums Fernsehens. Die daraus entwickelten Fernsehnutzungsmotivtypen werden durch Kontrastanalysen zu psychologischen und medienbezogenen Konstrukten in Bezug gesetzt.

In seiner Ergebnisdarstellung kann Michal Doh den Bildungs- und damit Einkommens- sowie Alterseinfluss in der Mediennutzung nachweisen. Die größte Heterogenität konstatiert er für die Altersgruppe der 70 bis 79 Jährigen. Weiterhin verweisen seine Ergebnisse ein mit zunehmendem Alter ansteigendes Informationsbedürfnis auch bei fernseh- und unterhaltungsaffinen Rezipienten, womit Doh die von Frank und Klinger 1987 aufgestellte These des Unterhaltungsslaloms im höheren Lebensalter widerlegt sieht. 
Im Ergebnisteil zur ILSE-Studie gelingt es dem Autor zu eruieren, welche individuelle Lebenslagen, Persönlichkeitsmuster und Altersprozesse sich hinter der in der Langzeitstudie Massenkommunikation (MK) offengelegten heterogenen Mediennutzungsformen älterer Menschen verbergen. In der durch eine Clusteranalyse erstellten Typologie zum Fernseherleben identifizierte Doh fünf typische Motivgruppen anhand der Grundorientierungen Information und Unterhaltung. Das Cluster «Die Durchschnittlichen» (1/3 des Samples) nutzen das Fernsehen gleichermaßen zur Information und Unterhaltung. Währenddessen ist für «Die distanzierten Informationsasketen» die Funktion des Fernsehens als Gebrauchsmedium zur Informationsgewinnung zentral. «Die Habituellen» kennzeichnen Spaß und Entspannung als dominierendes Nutzungsmotiv. Für «Die involvierten Unterhaltungsorientierten» hat das Fernsehen neben Information und Unterhaltung auch eine wichtige Orientierungsfunktion. Eine vergleichsweise starke Bindung zum Fernsehen zeichnet «Die Involvierten Eskapisten» (8\%) aus. Übergreifend zeigte sich in allen Clustern ein ausgeprägtes Interesse an Gesundheitsthemen, wobei dieses hinsichtlich physischem und psychischem Gesundheitszustand sowie kognitiven Einschränkungen stark differiert.

Ein interessantes Ergebnis der Analysen ist, dass trotz intensiver Nutzung und hoher Bindung an das Fernsehen in der Zielgruppe ein eher kritischer Blick auf das Medium bleibt. Nur 50 Prozent der Senioren zeigen eine moderate Zufriedenheit mit dem Medium, währenddessen die Zufriedenheit mit Radio, Zeitungen, Zeitschriften bei $90 \%$ liegt. Ein weiteres bemerkenswertes und gleichermaßen gesellschaftlich beunruhigendes Ergebnis der Analyse ist, dass etwa 60 Prozent der Senioren an keinerlei sozialen Aktivitäten teilnimmt. Der Aktionsradius im Alter ist auf das häusliche Umfeld bezogen, auch kulturelle, kirchliche und bildungsbezogene Aktivitäten spielen nur eine untergeordnete Rolle. Im abschließenden sechsten Kapitel fasst der Autor die Ergebnisse aus beiden Analysen zusammen und stellt einen Bezug zum theoretischen Bezugsrahmen der Arbeit her. 
Mit seiner umfassenden Arbeit leistet Michael Doh, insbesondere in der gelungenen Verbindung von medienwissenschaftlichen und gerontologischen Erkenntnissen, einen wichtigen Beitrag zum Themenfeld «Altern und Medien». Seine Differenzierungen eröffnen nicht nur einen Blick auf die heterogene Lebens- und Erfahrungswelt älterer Menschen; sie bieten gleichsam Impulse und Anschlüsse für eine weiterführende Auseinandersetzung.

Eher problematisch ist hingegen die undifferenzierte Thematisierung des Mediums Fernsehen, insofern dieses selbst von anhaltenden Umbrüchen und Transformationsprozessen geprägt ist. Wenig nachvollziehbar ist überdies die ausschweifende und überwiegend deskriptive Ergebnisdarstellung seiner Analysen quantitativer Nutzungsstudien, deren notwendiges Substrat selbst im resümierenden Schlussteil der Arbeit nur in Ansätzen zum Vorschein gebracht wird. Gerade mit Blick auf das ambitionierte Vorhaben der Arbeit wären in der hier angekündigten Diskussion (anstelle neuerlicher Deskriptionen) Überlegungen im Hinblick auf die Ausdifferenzierung eines zunehmend bedeutsam werdenden Forschungs- und Handlungsfeldes aufschlussreicher.

Anna-Maria Kamin

\section{Literatur}

Sander, Uwe; Vollbrecht, Ralf. Kinder und Jugendliche im Medienzeitalter. Annahmen, Daten und Ergebnisse empirischer Medienforschung. Opladen: Leske + Budrich, 1987.

Frank, Bernward; Klingler, Walter. Die veränderte Fernsehlandschaft. Zwei Jahre ARD/ZDF-Begleitforschung zu den Kabelpilotprojekten.

Frankfurt am Main: Metzner, 1987. 